TU-HEP-TH-99/110

\title{
Associated production of Higgs bosons and heavy quarks at photon colliders
}

\author{
Jun-Yu Guo, Yi Liao \\ Department of Physics, Tsinghua University, Beijing 100084, P.R.China \\ Yu-Ping Kuang \\ China Center of Advanced Science and Technology (World Laboratory), Beijing 100080, P.R.China \\ Department of Physics, Tsinghua University, Beijing 100084, P.R.China
}

\begin{abstract}
The idea of fermion mass generation through Yukawa couplings between Higgs bosons and fermions may be tested by their associated production at future linear colliders ( LC ). We study the production mechanism in the minimal supersymmeric standard model at photon colliders that can be realized by the laser back-scattering technique at the LC. We find that the cross sections for the production of the light Higgs-top pair, heavy Higgs-bottom pair and pseudoscalr Higgs-bottom pair can reach the level of $\sim 1.0 \mathrm{fb}$ or higher for phenomenologically favoured values of the parameters in the Higgs sector. Since this mechanism is not affected by the appearance of Higgs resonances as in electron collisions, it may provide a more sensitive way to determine the Yukawa couplings between Higgs bosons and heavy quarks.
\end{abstract}

Keywords: Higgs boson, Yukawa coupling, photon collider PACS: 14.80Cp, 12.60.Jv, 12.15.Ff

to appear in Phys. Rev. D 
The Higgs particle that is assumed to trigger the spontaneous breakdown of electroweak symmetries in the standard model (SM) or its extensions has so far been experimentally evasive. The current negative result of direct search imposes a lower bound on its mass, $m_{H}>100 \mathrm{GeV}$ [1]. Yet, global fits of precision electroweak data favour a relatively light SM Higgs boson with $m_{H}<260 \mathrm{GeV}$ at the $95 \%$ confidence level [2]. In the minimal supersymmetric extension of the SM (MSSM), there are five Higgs particles, the lightest of which cannot exceed a mass of about $130 \mathrm{GeV}$ because of supersymmetry and upon including radiative corrections [3]. More generally, there are arguments from triviality and vacuum stability that the SM Higgs boson mass should be in the narrow range of $130 \mathrm{GeV}<m_{H}<180 \mathrm{GeV}$ if the scale of new physics is of the order of the Planck scale[4] and that the lightest Higgs boson in general supersymmetric models should be lighter than about $200 \mathrm{GeV}$ [5]. All this implies a no-loss discovery of Higgs particles at the current or proposed future high energy colliders if there exist any such particles in Nature.

While the Higgs particles will probably be first discovered at LEP2, Tevatron or the future LHC, the future linear collider (LC) will provide an almost unique place to explore their detailed properties due to its clean environment and high luminosity. The successful discovery of Higgs particles will certainly advance our understanding of electroweak symmetry breaking and the mass generation of weak gauge bosons. However, it is important to notice that this does not mean that the issue of fermion mass generation will then automatically be solved since the latter could in principle be due to a separate ( or an additional ) origin. To answer this question, it will be necessary to examine the Higgs-fermion interactions independently.

In the SM, fermions acquire mass through the Higgs mechanism and the Yukawa couplings. Since these couplings are proportional to the mass of fermions considered, only the Higgs-top coupling is phenomenologically interesting. There are several ways to test the coupling. For example, we may measure the effective $H \gamma \gamma$ or $H g g$ vertices ( $H$ for the SM Higgs boson, $\gamma$ the photon and $g$ the gluon ), which essentially count the number of heavy particles, or the decay width of $H \rightarrow t \bar{t}$ if this is kinematically allowed. If the Higgs boson is not heavy, the 
Higgs boson radiation off top quarks at the future LC will provide a promising mechanism to study the coupling $[6][7][8]$. ( For the same production mechanism at hadron colliders, see Ref.[9]. ) In the MSSM, however, this simple pattern of associated production becomes complicated[7][10][11]. The Yukawa couplings are modified by the mixing angle $\alpha$ in the CPeven sector of the neutral Higgs bosons and $\tan \beta$, the ratio of the two vacuum expectation values of the Higgs fields. It turns out that the top production will be suppressed while the bottom enhanced as compared to the SM case for phenomenologically favoured large values of $\tan \beta$. Furthermore, for associated Higgs-bottom production, the resonant structures of the Higgs bosons must be included due to the off-diagonal $A^{0} H^{0} Z$ and $A^{0} h^{0} Z$ interactions. ( Here $H^{0}$ and $h^{0}$ stand for the CP-even heavy and light Higgs bosons, and $A^{0}$ the CP-odd one. ) The production rates are then overwhelmingly dominated by these resonant structures, which make the extraction of Yukawa couplings very involved and challenging. Actually, in the resonant region the sensitivity to Yukawa couplings is largely reduced so that one generally has to appeal to the continuum region for the extraction of couplings 11. 12.

In this work we shall consider an alternative way to measure the Yukawa couplings at the LC; namely, the associated production in photon collisions,

$$
\gamma \gamma \rightarrow Q \bar{Q} \phi, Q=t, b, \phi=h^{0}, H^{0}, A^{0}
$$

The technique using back-scattering laser lights [13 makes this a possible option for linear colliders which has been included in all proposed projects for the future linear colliders. The advantages using photon-photon or photon-electron collisions for some processes involving Higgs production have been extensively explored in the literature 14 15. For the process at hand, the SM case has already been examined in Ref. [16]. It was found that the rate for associated Higgs-top production in photon collisions is comparable to that in electron collisions while the background is slightly better. In the supersymmetric case to be investigated here, we generally cannot expect a rate as large as in electron collisions because of the resonant enhancement mentioned above. However, as far as the extraction of Yukawa couplings is concerned, one has to subtract the resonant region so that the effective rate is much smaller in electron collisions, 
while in photon collisions our processes will not be contaminated by similar resonances.

Let us first specify the Yukawa interaction between the generic Higgs boson $\phi$ and the fermion $\psi$,

$$
\mathcal{L}_{Y}=-\frac{m_{f}}{v} \bar{\psi}\left(a+i b \gamma_{5}\right) \psi \phi
$$

where $m_{f}$ is the fermion mass and $v=2^{-1 / 4} G_{F}^{-1 / 2}$. Our subsequent calculation applies to other models as well although we are mainly interested in phenomenology in the SM and the MSSM. The values for $a$ and $b$ are

$$
\mathrm{SM}: \quad \phi=H, a=1, b=0 ;
$$

$$
\begin{aligned}
\text { MSSM : } & \phi=h^{0}, a=\left\{\begin{array}{ll}
+\cos \alpha / \sin \beta, & \text { for up }- \text { type fermions } \\
-\sin \alpha / \cos \beta, & \text { for down }- \text { type fermions }
\end{array}, b=0,\right. \\
\phi & =H^{0}, a=\left\{\begin{array}{ll}
+\sin \alpha / \sin \beta, & \text { for up }- \text { type fermions } \\
+\cos \alpha / \cos \beta, & \text { for down }- \text { type fermions }
\end{array}, b=0,\right. \\
\phi & =A^{0}, a=0, b=\left\{\begin{array}{ll}
-\cot \beta, & \text { for up }- \text { type fermions } \\
-\tan \beta, & \text { for down }- \text { type fermions }
\end{array} .\right.
\end{aligned}
$$

In the MSSM, we have four Higgs masses and two angles $\alpha, \beta$. Supersymmetry imposes constraints on them so that only two of them are independent, which we shall choose to be $m_{A^{0}}$ and $\tan \beta$. These constraints are modified by radiative corrections. For the numerical analysis to be presented later on, we have included the contributions up to two loops in the effective potential approach [17].

The Feynman diagrams for the process $\gamma\left(k_{1}\right) \gamma\left(k_{2}\right) \rightarrow f\left(p_{1}\right) \bar{f}\left(p_{2}\right) \phi(q)$ are shown in Fig. 1, from which we obtain the amplitude,

$$
\begin{aligned}
\mathcal{A}= & +e^{2} Q_{f}^{2} \frac{m_{f}}{v} \bar{u}\left(p_{1}\right) \mathcal{A}_{\mu \nu} v\left(p_{2}\right) \epsilon^{\mu}(1) \epsilon^{\nu}(2), \\
\mathcal{A}_{\mu \nu}= & +Y\left(\not p_{1}+\not q+m_{f}\right) \gamma_{\mu}\left(-2 p_{2 \nu}+\not k_{2} \gamma_{\nu}\right)\left(D_{2,2} D_{1}\right)^{-1} \\
& +\left(2 p_{1 \mu}-\gamma_{\mu} \not k_{1}\right) Y\left(-2 p_{2 \nu}+\not k_{2} \gamma_{\nu}\right)\left(D_{1,1} D_{2,2}\right)^{-1} \\
& +\left(2 p_{1 \mu}-\gamma_{\mu} \not k_{1}\right) \gamma_{\nu}\left(-\not p_{2}-\not q+m_{f}\right) Y\left(D_{1,1} D_{2}\right)^{-1} \\
& +\left(k_{1} \leftrightarrow k_{2}, \mu \leftrightarrow \nu\right),
\end{aligned}
$$

where $Q_{f}$ is the charge of the fermion, $\epsilon(i)(i=1,2)$ are the photon polarization vectors, and

$$
Y=a+i b \gamma_{5}, \quad D_{i, j}=-2 k_{i} \cdot p_{j}, D_{i}=m_{\phi}^{2}+2 q \cdot p_{i}
$$

The gauge invariance and the Bose symmetry with respect to the photons are explicit in the above expression. 
To obtain the total cross section $\sigma$, we should take the convolution of the cross section $\hat{\sigma}$ for the subprocess with the photon luminosity spectrum $F(x)$,

$$
\sigma(s)=\int_{x_{\min }}^{x_{\max }} d x_{1} \int_{x_{\min } x_{\max } / x_{1}}^{x_{\max }} d x_{2} F\left(x_{1}\right) F\left(x_{2}\right) \hat{\sigma}\left(x_{1} x_{2} s\right)
$$

where $s$ is the c.m. energy squared for the parent electrons and the subprocess occurs effectively at $\hat{s}=x_{1} x_{2} s$. Here, $x_{i}$ are the fractions of the parent electrons' energies carried by the photons. For photons produced in the laser back-scattering technique, we have,

$$
\begin{aligned}
& F(x)=\frac{1}{D(\xi)}\left[1-x+\frac{1}{1-x}-\frac{4 x}{\xi(1-x)}+\frac{4 x^{2}}{\xi^{2}(1-x)^{2}}\right], \\
& D(\xi)=\left(1-\frac{4}{\xi}-\frac{8}{\xi^{2}}\right) \ln (1+\xi)+\frac{1}{2}+\frac{8}{\xi}-\frac{1}{2(1+\xi)^{2}}, \\
& x_{\max }=\frac{\xi}{1+\xi}, \xi=\frac{4 E_{0} \omega_{0}}{m_{e}^{2}},
\end{aligned}
$$

where $E_{0}$ and $\omega_{0}$ are the incident electron and laser light energies. To avoid unwanted $e^{+} e^{-}$ pair production from the collision between the incident and back-scattered photons, we should not choose too large $\omega_{0}$. This constrains the maximum value for $\xi$ to be $\xi=2(1+\sqrt{2})$. The minimum value for $x$ is then determined by the production threshold,

$$
x_{\min }=\frac{\hat{s}_{\min }}{x_{\max } s}, \hat{s}_{\min }=\left(2 m_{f}+m_{\phi}\right)^{2} .
$$

Our numerical results are shown in Figs. 2-5. The basic input parameters are, $\alpha=1 / 128$, $m_{W}=80.33 \mathrm{GeV}, m_{Z}=91.187 \mathrm{GeV}, m_{t}=174 \mathrm{GeV}$ and $m_{b}=5 \mathrm{GeV}$. We choose $\mu=A_{t}=$ $A_{b}=0$, and $\tilde{m}=1 \mathrm{TeV}$ for the generic squark mass. In the Higgs sector of the MSSM, we take $m_{A^{0}}$ and $\tan \beta$ as two independent parameters as mentioned above. To better understand our results for production rates, let us first present in Fig. 2 the reduced couplings squared $a_{h^{0}}^{2}, H^{0}$ and the masses $m_{h^{0}}, H^{0}$ of the CP-even Higgs bosons as functions of $m_{A^{0}}$ at $\tan \beta=3$ and 30 . We observe the following features from the panel (a). First, for fixed $\tan \beta$ and fermion type, the sum of $a_{h^{0}}^{2}$ and $a_{H^{0}}^{2}$ is correspondingly fixed to be $\sin ^{-2} \beta$ or $\cos ^{-2} \beta$ for up- or down-type fermions. Therefore, whenever the reduced coupling decreases for the heavy Higgs boson $H^{0}$, it increases for the light one $h^{0}$, and vice versa. For large values of $\tan \beta$, the down-type fermion couplings are enhanced while the up-type ones suppressed as compared to the SM case. Second, 
the relative importance of the $H^{0}$ and $h^{0}$ couplings to the same fermion is determined by $\tan \alpha$ which in turn depends on $\tan \beta$ and $m_{A^{0}}$. For the parameters considered here, $a_{h^{0}}^{2}>a_{H^{0}}^{2}$ for down-type fermions and $a_{h^{0}}^{2}<a_{H^{0}}^{2}$ for up-type fermions when $m_{A^{0}}$ is relatively small. The circumstance is reversed as $m_{A^{0}}$ goes up. Third, in the limit of large $m_{A^{0}}, a_{h^{0}}^{2}$ approaches one for both type of fermions, and $a_{H^{0}}^{2}$ becomes effectively the same as $b_{A^{0}}^{2}$. In this same limit, as shown in the panel (b), $m_{H^{0}}$ also becomes the same as $m_{A^{0}}$ while $m_{h^{0}}$ is saturated by its upper limit.

In Fig. 3 we plot the cross sections against the c.m. energy of the subprocess for the $t \bar{t} \phi$ ( panel (a) ) and $b \bar{b} \phi$ ( panel (b) ) production. It is clear that increasing the energy does not gain much in cross sections once we are well above the production threshold. This is especially true in the case of $b$ production, where the rate actually decreases as the energy goes up from $500 \mathrm{GeV}$. At photon colliders using back-scattering laser lights the produced photons are not monochromatic but constitute a continuous spectrum. This effect is taken into account in Figs. 4 and 5 at two typical c.m. energies of the parent electrons. The behaviour of cross sections follows closely the pattern of the reduced couplings squared as we have just discussed, up to modification factors introduced by the scattering amplitude and the phase space. Let us first go over the top pair production. When $\tan \beta$ is not too large, the associated $h^{0}$ production always dominates, with a typical rate of about $1.0 \mathrm{fb}$. The rate for $H^{0}$ depends on the detail of the parameters $\tan \beta$ and $m_{A^{0}}$. For small values of $m_{A^{0}}$, it is comparable to the rate for $h^{0}$ or even dominates when $\tan \beta$ is large enough. The pseudoscalar $A^{0}$ is the most difficult to produce. However, this hierarchy in cross sections is almost reversed in the associated bottomHiggs production which is phenomenologically relevant only for large enough tan $\beta$. Here we see that the production rate of the pseudoscalar $A^{0}$ is always the largest and can reach several $\mathrm{fb}$ while the $h^{0}$ production is competitive only for relatively small $m_{A^{0}}$. The behaviour of $H^{0}$ in the large $m_{A^{0}}$ limit becomes identical to that of $A^{0}$ because their couplings, masses and the scattering amplitudes squared approach each other in this limit. Also shown in the panel (a) of Figs. 4 and 5 is the SM model result for the $t \bar{t} H$ production. It is clear that in the decoupling 
limit of large $m_{A^{0}}$ the $h^{0}$ production rate approaches correctly that of the SM Higgs $H$ with the same mass as $h^{0}$, which is about $98 \mathrm{GeV}$ for $\tan \beta=3$ and $112 \mathrm{GeV}$ for $\tan \beta=30$. Comparing Figs. 4 and 5 we realize that only the heavy $A^{0}$ and heavy $H^{0}$ production with top pairs is considerably enhanced as the energy changes from $1 \mathrm{Tev}$ to $2 \mathrm{TeV}$, which is consistent with the pattern observed in Figs. 2(a) and 3(a).

In summary, we have considered the associated production of Higgs bosons and heavy quarks at the LC operating in the photon mode which may be used to measure their Yukawa couplings. For the SM case we reproduced the previous result that the production rate is comparable to that in the parent electron collisions. In the more complicated case of the MSSM, the efficiency of extracting Yukawa couplings becomes somewhat vague in the associated bottomHiggs production of electron collisions due to the contamination by the Higgs resonances. Instead, we proposed to determine these couplings through photon collisions where no such resonances are involved. We found that the production rate for $t \bar{t} h^{0}$ is as large as $1.0 \mathrm{fb}$ while $b \bar{b} H^{0}$ and $b \bar{b} A^{0}$ can be equally or more copiously produced for phenomenologically favoured large values of $\tan \beta$. This amounts to about 500 events or more for an annually integrated luminosity of $500 \mathrm{fb}^{-1}$ at the TESLA. In this manner, the measurement at photon colliders may provide us a global picture of the Yukawa couplings between the neutral Higgs bosons and the heavy quarks. We also found that a high luminosity is more essential than a high c.m. energy for this purpose. Finally, we should mention that we have considered only the lowest order contribution which may receive potentially large radiative corrections especially for the bottom pair production in the case of large $\tan \beta$.

Acknowledgements. Y.L. would like to thank M. Spira and P. M. Zerwas for helpful discussions concerning the effect of Higgs resonances on the associated bottom-Higgs production in electron collisions. He is also grateful to M. Spira for providing us the Fortran code 18 to compute the Higgs masses and couplings based on the work in Ref. 117.

\section{References}


[1] ALEPH Collaboration, hep-ex/9908016; DELPHI Collaboration, CERN-EP-99006; L3 Collaboration, Phys. Lett. B461, 376(1999); OPAL Collaboration, hepex/9908002.

[2] LEP Collaborations, CERN-EP-99-015.

[3] Y. Okada, M. Yamaguchi and T. Yanagida, Prog. Theor. Phys. 85, 1(1991); H. Haber and R. Hempfling, Phys. Rev. Lett. 66, 1815(1991); J. Ellis, G. Ridolfi and F. Zwirner, Phys. Lett. B257, 83(1991); R. Barbieri and M. Frigeni, ibid, B258, 395(1991).

[4] M. Sher, Phys. Lett. B317, 159(1993); G. Altarelli and G. Isidori, ibid, B337,341(1994); J. Casa, J. Espinosa and M. Quiros, ibid, B382, 374(1996); T. Hambye and K. Riesselmann, Phys. Rev. D55, 7255(1997).

[5] M. Drees, Int. J. Mod. Phys. A4, 87(1989); U. Ellwanger, M. Rausch de Traubenberg and C. A. Savoy, Nucl. Phys. B492, 21(1997); J. Espinosa and M. Quiros, Phys. Rev. Lett. 81, 516(1998).

[6] K. J. F. Gaemers and G. J. Gounaris, Phys. Lett. B77, 379(1978); A. Djouadi, J. Kalinowski and P. M. Zerwas, Mod. Phys. Lett. A7, 1765(1991).

[7] A. Djouadi, J. Kalinowski and P. M. Zerwas, Z. Phys. C54, 255(1992).

[8] For QCD corrections to $e^{+} e^{-} \rightarrow t \bar{t} H$ in the SM, see: S. Dawson and L. Reina, Phys. Rev. D57, 5851(1998); ibid, D59, 054012(1999); S. Dittmaier, M. Kraemer, Y. Liao, M. Spira and P. M. Zerwas, Phys. Lett. B441, 383(1998). Simulations including backgrounds are considered by: S. Moretti, ibid, B452, 338(1999); H. Baer, S. Dawson and L. Reina, Phys. Rev. D61, 013002(2000).

[9] For a partial list of associated Higgs and heavy quark production at hadron colliders, see for example: R. Raitio and W. W. Wada, Phys. Rev. D19, 941(1979); J. N. Ng and P. Zakarauskas, ibid, D29, 876(1984); Z. Kunszt, Nucl. Phys. B247, 339(1984); J. F. Gunion, H. E. Haber, F. E. Paige, W.-K. Tung and S. Willenbrock, ibid, B294, 621(1987); D. A. Dicus and S. Willenbrock, Phys. Rev. D39, 751(1989); J. F. Gunion, 
Phys. Lett. B261, 510(1991); W. J. Marciano and F. E. Paige, Phys. Rev. Lett. 66, 2433(1991); Z. Kunszt, S. Moretti and W. J. Stirling, Z. Phys. C74, 479(1997); D. Dicus, T. Stelzer, Z. Sullivan and S. Willenbrock, Phys. Rev. D59, 094016(1999); J. Dai, J. F. Gunion, R. Vega, Phys. Lett. B345, 29(1995); ibid, B387, 801(1996); J. L. Diaz-Cruz, H.-J. He, T. Tait, C. P. Yuan, Phys. Rev. Lett. 80, 4641(1998); C. Balazs, J. L. Diaz-Cruz, H.-J. He, T. Tait, C. P. Yuan, Phys. Rev. D59, 055016(1999); M. Carena, S. Mrenna, C. E. M. Wagner, Phys. Rev. D60, 075010(1999).

[10] S. Dawson and L. Reina, Phys. Rev. D60, 015003(1999).

[11] S. Dittmaier, M. Kraemer, Y. Liao, M. Spira and P. M. Zerwas, hep-ph/0002035, to appear in Phys. Lett. B.

[12] M. Spira and P. M. Zerwas, private communications.

[13] I. Ginzburg, G. Kotkin, V. Serbo and V. Telnov, Pizma ZhETF 34, 514(1981); JETP Lett. 34, 491(1982); Nucl. Instr. and Meth. 205, 47(1983); I. Ginzburg, G. Kotkin, S. Panfil, V. Serbo and V. Telnov, Nucl. Instr. and Meth. 219, 5(1984).

[14] For $\gamma \gamma$ collisions, see for example: G. V. Jikia and Yu. F. Pirogov, Phys. Lett. B283, 135(1992); G. V. Jikia, Nucl. Phys. B412, 57(1994); L. Z. Sun, Y. Y. Liu, Phys. Rev. D54, 3563(1996); I. F. Ginzburg, M. Krawczyk and P. Osland, hep-ph/9909455.

[15] For e collisions, see for example: D. A. Dicus and W. W. Repko, Phys. Rev. D53, 3616(1996); E. Gabrielli, V. A. Ilyin and B. Mele, ibid, D56, 945(1997) and Erratum, D58, 119902(1998); U. Cotti, J. L. Diaz-Cruz and J. J. Toscano, Phys. Lett. B404, 308(1997); Y. Liao and W. W. Repko, Phys. Rev. D57, 6998(1998).

[16] E. Boos, I. Ginzburg, K. Melnikov, T. Sack and S. Shichanin, Z. Phys. C56, 487(1992); K. Cheung, Phys. Rev. D47, 3750(1993).

[17] M. Carena, M. Quiros and C. E. M. Wagner, Nucl. Phys. B461, 407(1996); H. E. Haber, R. Hempfling and A. H. Hoang, Z. Phys. C75, 539(1997); S. Heinemeyer, W. Hollik and G. Weiglein, Phys. Rev. D58, 091701 (1997). 
[18] A. Djouadi, J. Karlinowski and M. Spira, Comput. Phys. Commun. 108, 56(1998).

\section{Figure Captions}

Fig. 1 Feynman diagrams for the subprocess $\gamma \gamma \rightarrow f \bar{f} \phi$. Crossing diagrams are not shown.

Fig. 2 Reduced couplings squared $a_{h^{0}, H^{0}}^{2}\left(\right.$ panel (a)) and masses $m_{h^{0}, H^{0}}$ ( panel (b)) as functions of $m_{A^{0}}$ for $\tan \beta=3$ ( solid ) and 30 ( dashed ). $b_{A^{0}}^{2}$ is fixed to be $\tan ^{2} \beta$ or $\cot ^{2} \beta$ which is not shown.

Fig. 3 Cross sections as functions of the c.m. energy of monochromatic photons for $m_{A^{0}}=150$ $\mathrm{GeV}, \tan \beta=3$ ( solid ) and 30 (dashed ): (a) $t \bar{t} \phi$ production; (b) $b \bar{b} \phi$ production.

Fig. 4 Cross sections as functions of $m_{A^{0}}$ in the laser back-scattering technique at the electrons' energy $\sqrt{s}=1 \mathrm{TeV}$ for $\tan \beta=3$ ( solid ) and 30 ( dashed ): (a) $t \bar{t} \phi$ production; (b) $b \bar{b} \phi$ production. The long-dashed curve in the panel (a) stands for the SM Higgs for which $m_{A^{0}}$ should be understood as $m_{H}$.

Fig. 5 Same as Fig. 4 , but at $\sqrt{s}=2 \mathrm{TeV}$.
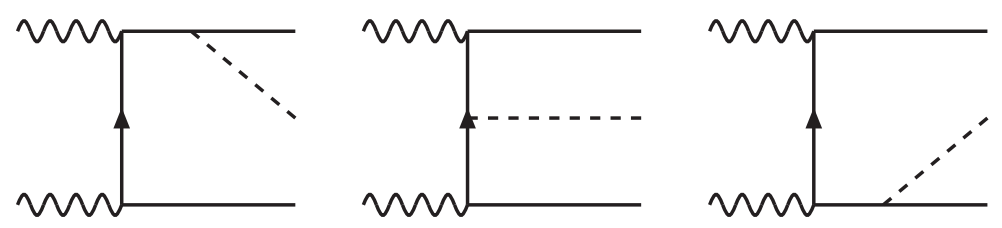

Figure 1 

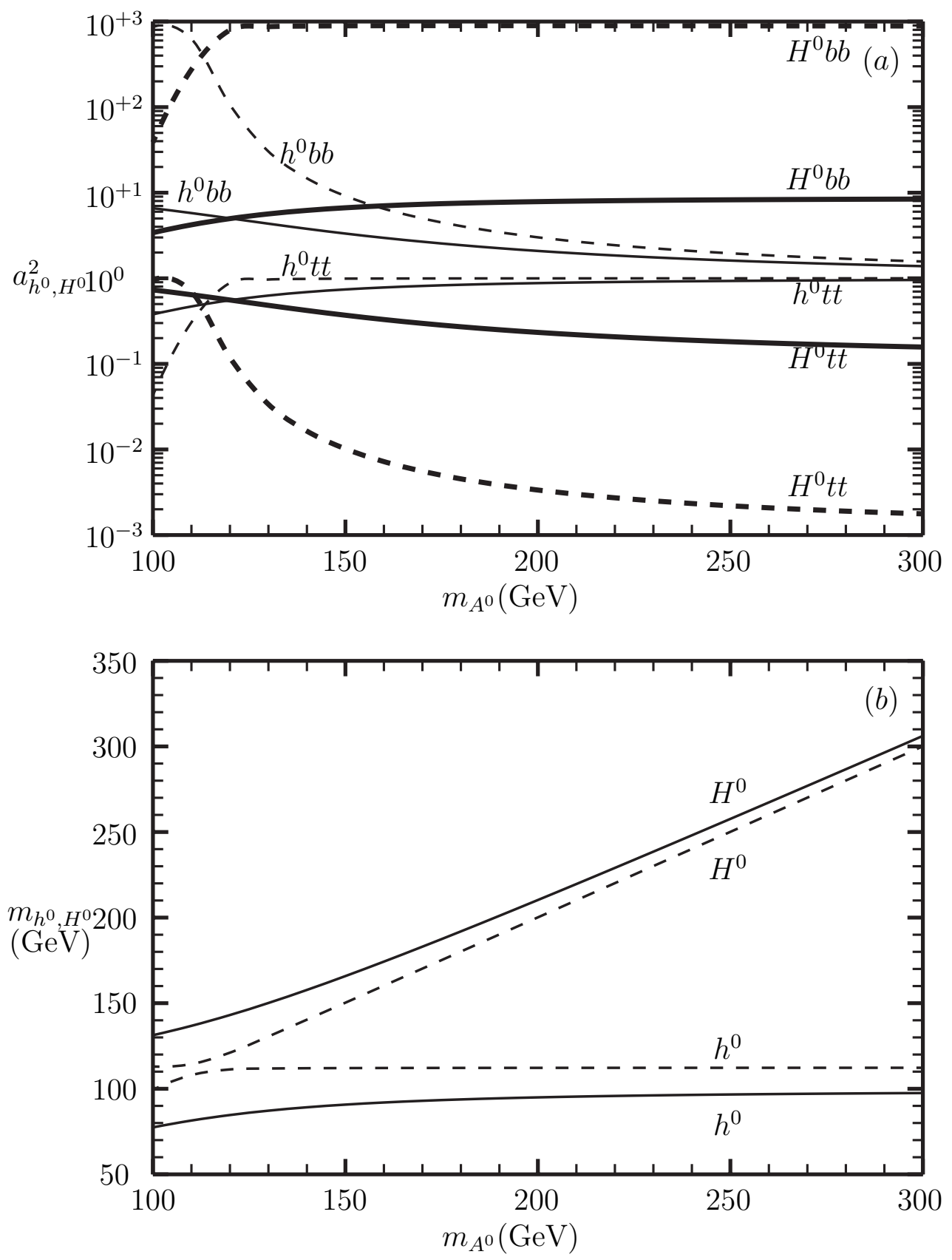

Figure 2 

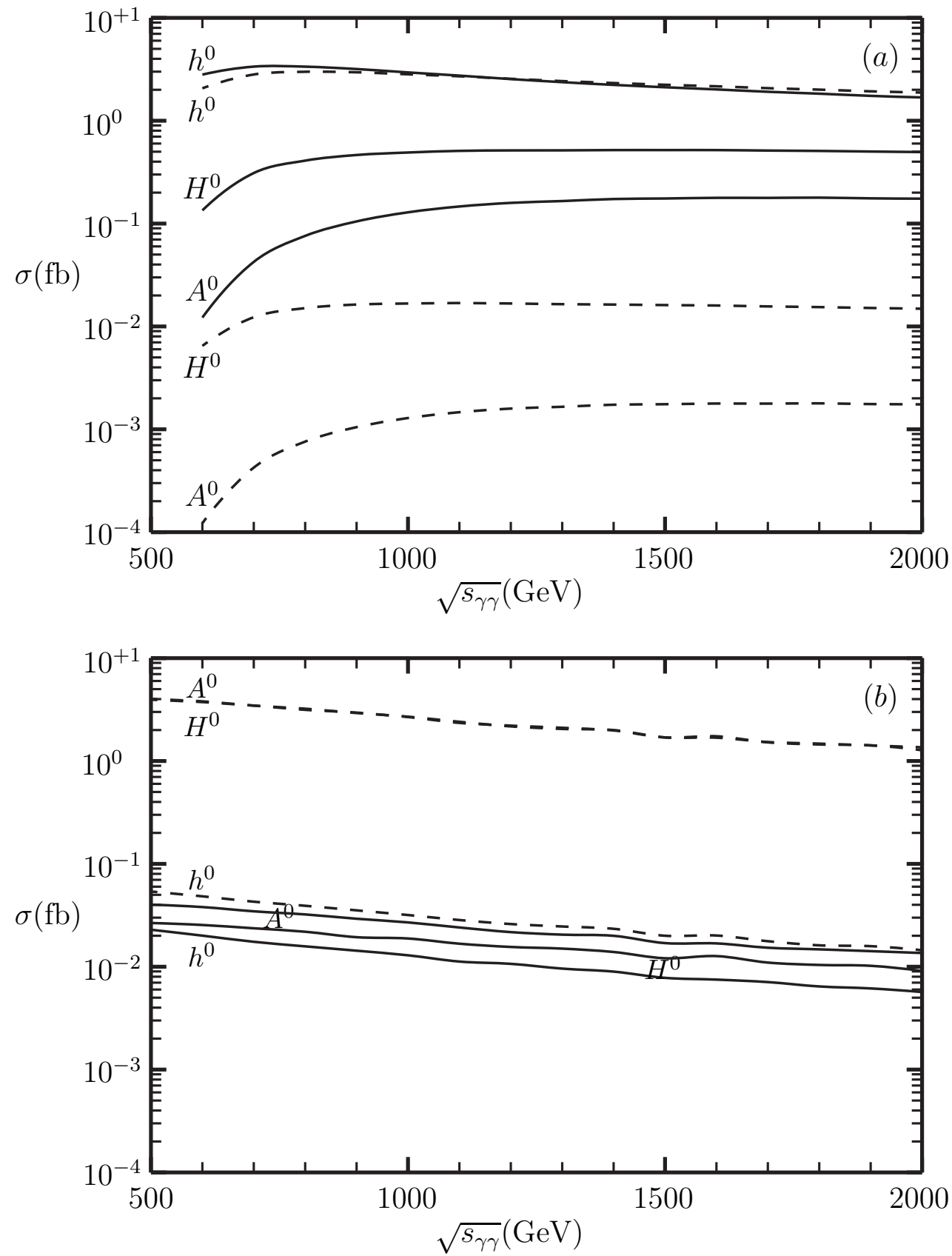

Figure 3 

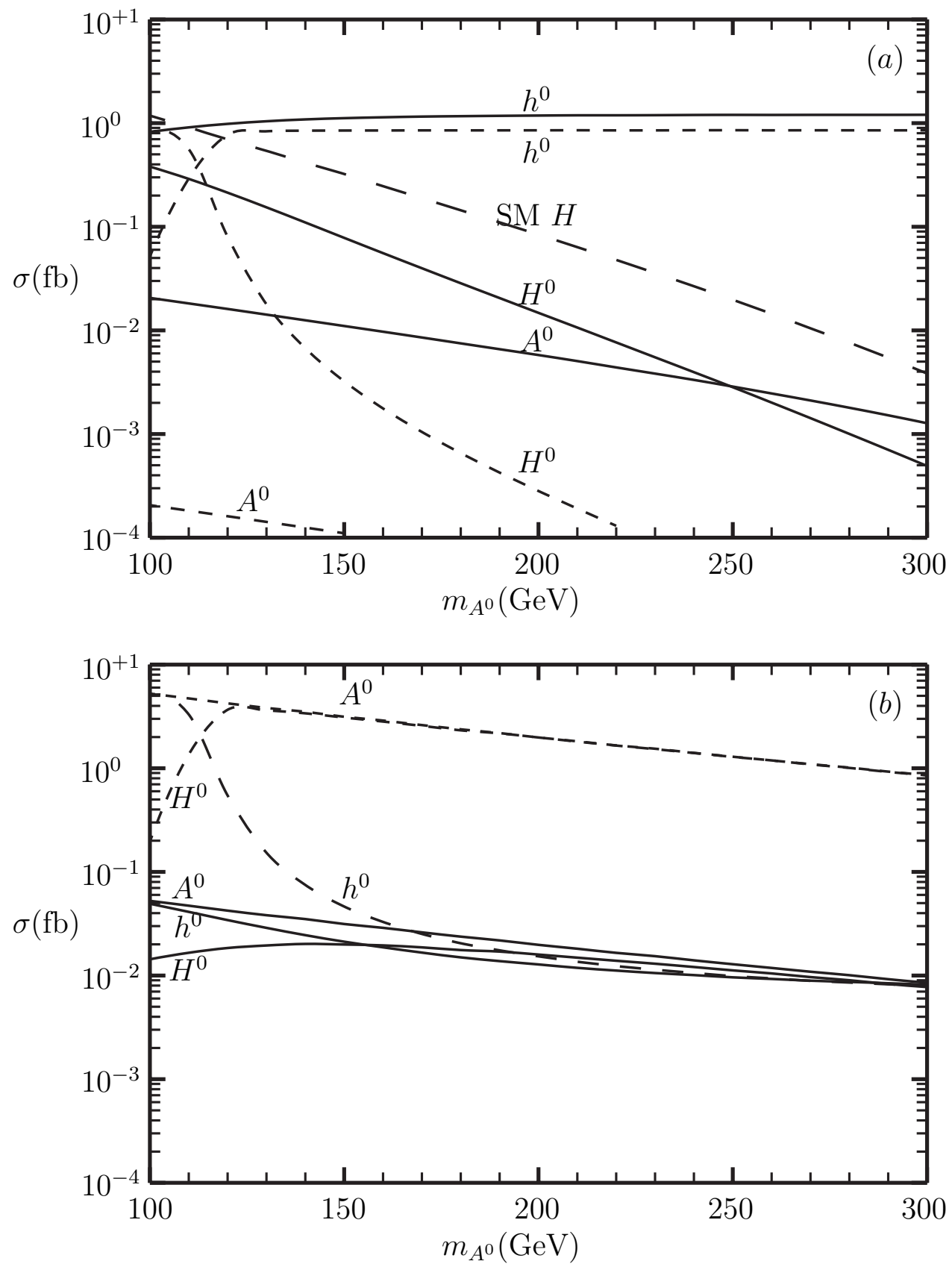

Figure 4 

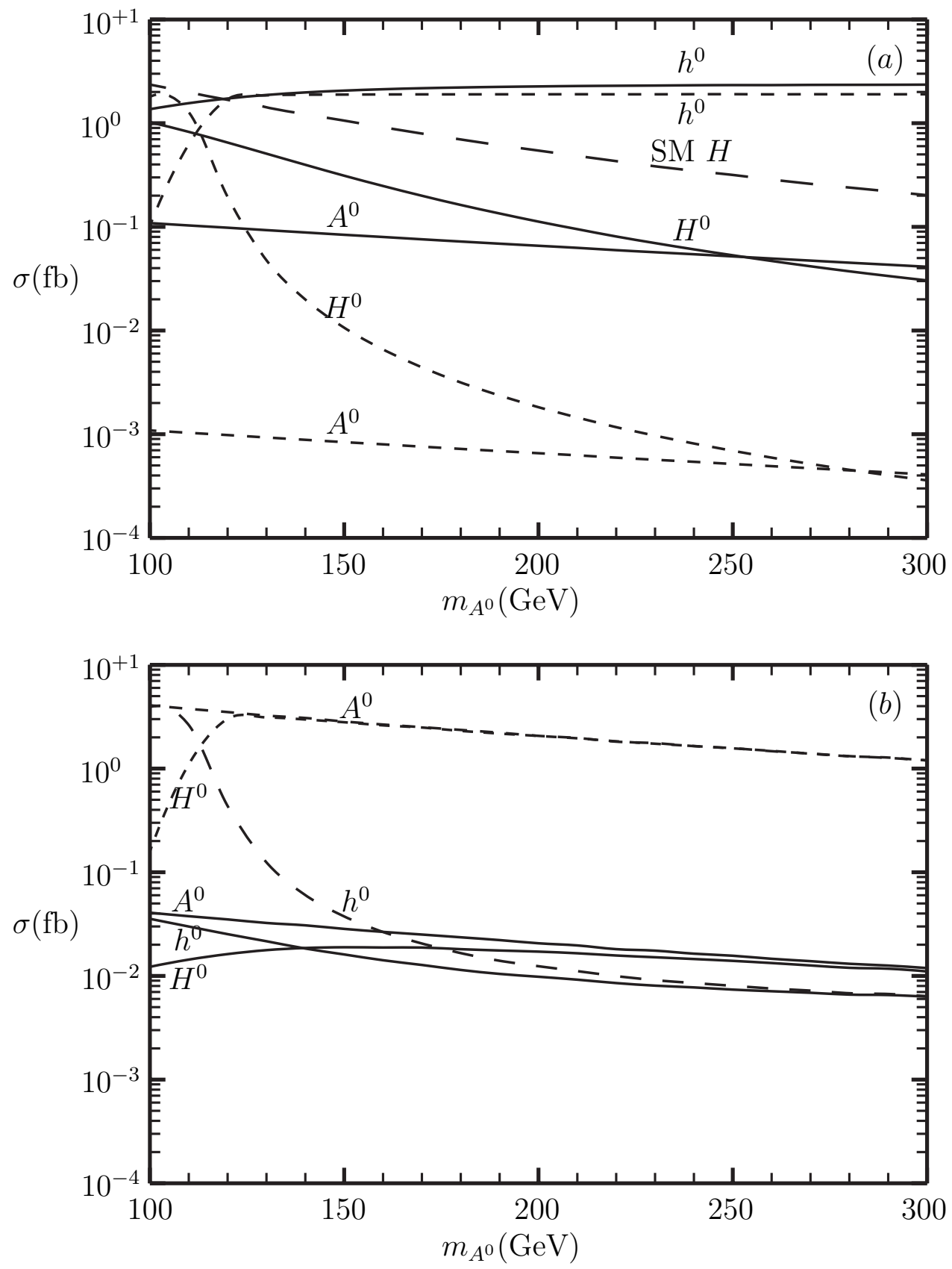

Figure 5 